[17] M. Seelemann. Die Galtbehandlung, ein erfolgversprechendes und segens. reiches Arbeitsgebiet für den praktisehen Tierarzt. Tierärztl. Rdsch., $n^{\circ} 34,577 ; n^{0} 35,593$ (1932).

\title{
LES MASTITES DE LA VACHE (1)
}

par

M. le Docteur F. C. MINETT,

Research Institute in Animal Pathology, Royal Veterinary College, London.

On a essayé dans ce rapport, de présenter de manière succinete, mais critique, quelques-unes de nos connaissances les plus importantes sur la mastite bovine. Une attention particulière a été accordée aux problèmes du diagnostic et du contrôle de la forme streptococcique chronique de la maladie.

\section{FORMES DE LA MASTITE.}

Le terme "mastite" comprend un certain nombre d'états morbides provoqués par différentes espèces de bactéries. De multiples essais ont été tentés pour classer ces états, mais on peut admettre que la seule classification réellement satisfaisante doit être basée sur l'étiologie, et c'est la méthode qui a été adoptée dans le présent rapport.

\section{STREPTOCOCCUS MASTITIS.}

Il est bien connu que la grande majorité des infections de la mamelle sont causées par les streptocoques et que, dans la forme la plus commune (celle appelée " gelber Galt "), le début est insidieux et la cause essentiellement persistante [15]. Les vétérinaires sont si familiarisés avec les signes cliniques de cet état que je n'ai pas l'intention de m'y arrêter plus longtemps.

Une seconde forme clinique est celle dans laquelle la maladie affecte une évolution aiguë dès le début, aboutissant à la perte rapide d'un quartier qui auparavant paraissait sain. Bien que cette variété soit beaucoup moins fréquente que la précédente, on sait qu'elle cause des pertes considérables [9].

Une telle différenciation de la mastite streptococcique en forme chronique et en forme aiguë, bien qu'elle corresponde à la classification bactériologique ci-dessous, est peu rigoureuse du point de vue clinique. Ainsi la mastite chronique, au cours d'une évolution prolongée; présente assez souvent des phases aiguës, tandis que dans la forme plus aiguë les symptômes sont par moments comparativement bénins, et, dans ce cas, l'infection peut

(1) Rapport présenté à l'Office International des Epizooties, publié dans le Bulletin de l'Office International des Epizooties, 1932, 6, 124. 
ne persister que peu de temps et ne causer qu'une perte partielle de la fonction.

Bactériologie. - a) Les streptocoques qui provoquent la maladie chronique appartiennent à un groupe parfaitement défini, pour lequel la désignation "Streptococcus agalactice" est devenue d'un usage général dans l'Europe centrale. C'est sans doute le même organisme qui a été originairement isolé, en 1887, par NocARD et Molleread dans la "mammite contagieuse de la vache". On peut faire à la création de désignations comme celles de Streptococcus agalactice les mêmes objections qu'à celles de Streptococcus scarlatinae, Streptococcus bovis, etc. Il est possible, dans un sens étroit, de s'opposer à l'utilisation d'un terme comme Streptococcus agalactice (même si cette désignation est associée au nom d'un expérimentateur particulier), parce qu'il n'y a aucune raison pour le réserver à l'un seulement des groupes de streptocoques qui provoquent la mastite. L'utilisation habituelle de ce terme pour le groupe de streptocoques que l'on rencontre le plus souvent dans la mastite ne peut être défendue qu'en raison de sa commodité et parce qu'elle est généralement employée par nombre d'expérimentateurs.

Les principaux caractères culturaux et biochimiques de ce groupe n'ont pas besoin d'être passés en revue. Dans la règle, les propriétés hémolytiques ne sont pas très marquées, bien que les souches varient sous ce rapport. Quelques souches présentent l'hémolyse du type $\beta$ de Brown autour des colonies profondes sur gélose-sang en plaques, bien que les zones soient étroites et les bords généralement flous. D'autres souches présentent l'hémolyse de la var a ou $\alpha^{\prime}$. Certaines des souches les plus hémolytiques produisent des quantités appréciables d'hémolysine après culture pendant huit heures dans le bouillon sérumisé à 10 p. 100 . SeELEMANN [13] a récemment décrit des souches "atypiques" de $S$. agalactia, mais on ne peut encore admettre quil existe des raisons suffisantes pour justifier cette nouvelle différenciation.

b) Les streptocoques responsables des formes plus aiguës de mastite, mentionnées plus haut, ont fait l'objet de peu d'études. Un travail récent, publié en Grande-Bretagne [6] [3] a démontré que beaucoup de ces streptocoques peuvent être classés dans un même groupe et possèdent des caractères culturaux et biochimiques qui les distinguent de $S$. agalactice. Il y a des raisons de penser que jusqu'ici ces streptocoques ont été parfois confondus avec S. lactis, encore qu'ils présentent de nombreux earactères différentiels.

c) Une troisième variété de streptocoques de la mastite a été isolée, qui diffère de $S$. agalactice, entre autres caractères, en ce qu'elle est nettement hémolytique et hautement virulente pour les 
petits animaux d'expérience. D'autre part, cette variété ne présente pas de point de différence importante avec Streptococcus pyogenes de l'homme. Lorsqu'ils sont isolés pour la première fois, ces streptocoques possèdent parfois des capsules délimitées, et pour cette raison les colonies sur gélose-sang développées dans une atmosphère humide sont liquéfiées et étalées. Des organismes semblables ayant été rencontrés un certain nombre de fois en relation avec des angines épidémiques, le terme de Streptococcus epidemicus est d'un usage fréquent pour les désigner, spécialement aux Etats-Unis. Cliniquement, il n'existe pas de moyens certains pour distinguer la mastite due à cet organisme de celle provoquée par S. agalactice.

\section{STAPHYLOCOCCUS MASTITIS.}

Les vaches sont souvent atteintes d'une variété de mastite, provoquée par des staphylocoques, dans laquelle on constate des troubles généraux graves et une forte tendance à la gangrène de la mamelle. La mortalité est élevée, mais des cas de guérison se produisent quelquefois à la suite de la séquestration des parties atteintes. Des streptocoques liquéfiant la gélatine, hémolytiques, se trouvent en nombre considérable dans la sécrétion, qui, dans le stade du début, consiste en un liquide clair, ressemblant à du sang hémolysé.

\section{BACT. PYOQENES MASTITIS.}

Bact. pyogenes est maintenant reconnu comme la cause la plus fréquente de la variété purulente de mastite connue en GrandeBretagne sous le nom de "summer mastitis »; en Allemagne et en France, sous celles de "Weideeuterseuche" et de " mammite d'herbage ". Les signes cliniques et épidémiologiques de cette maladie et les caractères bactériologiques de l'organisme causal étant bien connus, on s'abstient de s'y arrêter plus longuement. (Voir la monographie de RoLLE [11].)

\section{MASTITES DUES A D'AUTRES BACTERIES.}

Une petite proportion des cas de mastite est due à des espèces bactériennes autres que celles ci-dessus mentionnées, les signes cliniques variant avec chacune d'entre elles. En dehors de la mastite tuberculeuse, l'infection de la mamelle peut être causée par Bact. coli et par les formes voisines, par Bact. pyocyaneus, Bact. necrosis, l'Actinomyces et certaines levures.

\section{RÉPARTITION ET IMPORTANCE ÉCONOMIQUE.}

Tous ceux qui sont familiarisés avec le sujet sont d'accord sur la vaste extension de la mastite et sur sa grande importance économique. Quelques essais ont été tentés pour évaluer la fréquence 
de la forme streptococcique chronique. La valeur de ces évaluations dépend naturellement du nombre et de la nature des observations, aussi bien que des méthodes utilisées pour diagnostiquer les infections latentes de la glande. Trois exemples suffiront pour démontrer la fréquence de la mastite streptococcique.

Pвöschold [10], étudiant, en 1928, la fréquence de la maladie en Poméranie, relève que, sur 7.809 vaches appartenant à des troupeaux ne comptant pas en moyenne moins de 50 animaux, 1.228 (soit $15,8 \%$ ) étaient infectées. Dans quelques troupeaux, la proportion des infectées atteignait $80 \%$.

Semlemann [13], dans sa précieuse monographie, rapporte qu'entre 1926 et 1931, il a examiné, en Allemagne, des échantillons de laits provenant de 7.691 vaches appartenant à 173 troupeaux ; 140 de ces troupeaux se trouvaient dans le Sleswig-Holstein. Parmi ces vaches, 2.539 (33 \%) étaient infectées, 3.694 (48 \%) étaient saines et $1.458(19 \%)$ étaient suspectes. Cette maladie était loin d'être rare dans les petits troupeaux de dix vaches ou moins.

A l'Institut de recherches du Royal Veterinary College de Londres, des données concernant 1.384 vaches, appartenant à vingt-einq troupeaux de la Grande-Bretagne, ont été recueillies entre 1928 et 1932. Streptococous mastitis était présent dans tous ces troupeaux, et 548 vaches $(39,5 \%)$ ont été trouvées infectées dans un quartier au moins de la mamelle ; la proportion, dans chaque troupeau, variait de 11 à $65 \%$. La présence de l'infection semble n'avoir aucun rapport avee la classe du troupeau.

Il ne faut pas s'imaginer, d'après ces chiffres, qu'il y ait une différence appréciable dans la fréquence de la maladie pour les trois régions mentionnées. D'abord, le nombre de troupeaux ainsi examinés en Grande-Bretagne est relativement faible et, en second lieu, la méthode d'investigation n'a pas été tout à fait la même dans les différents cas.

Un second facteur d'accroissement des pertes économiques dues à la mastite streptococcique est que sa fréquence augmente avec l'âge. Ainsi, l'infection est nettement plus répandue parmi les vaches au-dessus de cinq ans, c'est-à-dire chez les vaches qui atteignent leur capacité maximum de production laitière.

Quant à l'étendue des lésions chez chaque animal, SeELEmann observe que le pourcentage des vaches dont un seul quartier est atteint est relativement faible. L'examen de 1.416 quartiers chez 394 vaches infeetées démontre que 843 quartiers $(59,5 \%)$ contiennent les streptocoques de la mastite.

Avec la perte du lait, qui, dans l'ensemble, doit causer un dommage considérable, jl faut faire ressortir la dépréciation individuelle des animaux et le déchet excessif du troupeau. De plus, la qualité du lait sécrété par des vaches infectées est gravement compromise par suite des modifications de sa composition, la diminution du lactose et de la graisse, et en raison de la présence des résidus de la réaction, comme le sérum sanguin et les leucocytes. 


\section{LA PATHOGÉNIE de LA MASTITE.}

Les indications sur cette partie du sujet sont quelque peu confuses, de telle sorte que les considérations dogmatiques peuvent être écartées.

Dans le cas de mastite streptococcique chronique, on a suggéré l'idée que les organismes peuvent être apportés à la mamelle par la cireulation sanguine á la suite de l'ingestion d'un matériel infectieux. Ce point de vue n'est pas appuyé par des expériences dans lesquelles de grandes quantités de lait infectieux auraient été données par la bouche, et il n'est pas probable qu'une contamination massive des aliments par un tel lait se produise souvent dans la pratique.

Une hypothèse plus vraisemblable et entièrement en accord avec les faits d'observation est que l'infection est propagée par les mains du trayeur et qu'elle pénètre dans la glande par la voie du canal du trayon. La manière dont l'organisme s'installe dans la glande est discutée par KLIMmer et HaUPT dans leur admirable revue sur cette question. On peut supposer que la présence de fissures ou d'érosions de la peau du trayon près du méat favorisent l'infection, encore qu'on ait aussi supposé que les streptocoques peuvent pénétrer directement dans la glande par des ulcérations de toutes les parties du trayon. S'il est possible de provoquer l'invasion de la mamelle par l'injection, dans le canal du trayon, de lait contenant les streptocoques de la mastite, il est difficile d'atteindre ce résultat par les mulsions répétées avec des mains enduites de lait infectieux. C'est ainsi que l'on constate le fait surprenant que chez une vache un des quartiers de la mamelle peut être depuis longtemps infecté sans que les autres quartiers le soient. Il semble, sans que l'explication soit évidente, que certains quartiers soient considérablement plus résistants que d'autres. Des expériences dans lesquelles les extrémités des trayons ont été enduits de cultures appuient l'hypothèse que les quartiers diffèrent entre eux quant à leur susceptibilité, et la diversité des expressions cliniques dans les divers quartiers peut être rapportée à la même cause.

La dissémination de Bact. pyogenes mastitis par les mains du trayeur est fréquemment exclue. Pour eette raison, étant donné que les animaux au pâturage durant l'été peuvent être atteints en même temps, on a supposé que les mouches agissent comme agents transmetteurs. Le bien-fondé de cette explication n'est pas établi jusqu'ici.

En ce qui concerne la mastite staphylococcique et les formes plus aiguës de la mastite streptococcique, les faits d'observations ne sont pas en faveur de la notion que la maladie se propage par contagion. Apparemment, ces formes procèdent de quelque foyer préexistant de maladie dans l'organisme ou de quelque cause prédis- 
posante qui détermine une multiplication excessive des bactéries considérées comme des hôtes normaux de la mamelle. La première de ces alternatives semble plus probable pour expliquer l'origine de Bact. necrosis mastitis, et elle peut expliquer aussi en certains cas celle de Bact. pyogenes mastitis.

\section{LA MASTITE ET LA SANTÉ PUBLIQUE.}

Si l'on considère la fréquence de la mastite streptococcique et le fait qu'une grande quantité de lait est consommé à l'état cru, on peut admettre que les streptocoques ordinairement rencontrés dans la mastite ne sont pas dangereux pour l'homme. Cette constatation ne signifie pas que la mastite streptococcique n'a aucune importance pour la santé publique ; elle en a non seulement parce que la valeur nutritive du lait des vaches infectées est diminuée, mais parce que l'usage, dans l'alimentation de l'homme, du lait contenant effectivement du pus, est très regrettable.

Il est maintenant certain que la consommation d'un tel lait présente encore d'autres inconvénients. Comme on le sait, certaines maladies humaines autres que la tuberculose, par exemple la fièvre typhoïde, la dysenterie, etc., sont transmises à l'occasion par le lait. Des maladies dues aux streptocoques apportées à l'homme par le lait ont été ainsi observées : Aux Etats-Unis, de 1908 à 1926, selon Armstrong et Parran [1], il a été enregistré quatre-vingt-deux épidémies de fièvre scarlatine et d'angine septique transmises par le lait, avec 25.000 malades et 159 morts.

Relativement aux épidémies provoquées par le lait qui ont sévi en Grande-Bretagne vers la fin du siècle dernier, beaucoup d'apparences tendaient à désigner les vaches comme la source du mal. En conséquence, on suggéra l'idée que les foyers étaient imputables à une affection constitutionnelle des vaches, à laquelle on donna le nom de bovine scarletina. Cette opinion ne fut pas longtemps acceptée, et il semble que l'on possède maintenant une explication satisfaisante des faits. En 1911, SAVAGE [12] émit l'hypothèse que si la mastite est la source de la maladie à streptocoques chez l'homme, c'est parce que l'organisme en cause était d'origine humaine. Ce point de vue a été confirmé par les recherches poursuivies aux Etats-Unis depuis 1911. L'hypothèse explique des particularités communément observées dans les épidémies, par exemple leur début explosif, leur persistance et le grand nombre des personnes atteintes en même temps. Il a été possible de démontrer, dans un certain nombre de foyers, que les streptocoques isolés du lait des vaches suspectes étaient non seulement différents de ceux qui sont ordinairement associés à la mastite, mais qu'ils étaient en fait identiques à ceux isolés des malades humains. Dans le lait des vaches en gues- 
tion, le nombre des streptocoques est extrêmement élevé à certains moments, si bien que la présence d'une seule vache infectée peut être suffisante pour produire une contamination dangereuse du lait de mélange de tout un troupeau. De plus, la glande peut recéler les streptocoques pendant au moins plusieurs semaines, et les symptômes cliniques peuvent être si légers que la sécrétion n'est ordinairement pas écartée comme impropre à la consommation. Il y a donc de fortes probabilités pour que, du moins dans les grandes épidémies, la vache intervienne communément comme la source immédiate de l'infection. L'accord sur ce point n'empêche pas que certains foyers moins importants puissent être provoqués par une contamination directe du lait par les êtres humains.

Au cours des six dernières années, l'opinion scientifique concernant l'importance de la mastite pour la santé publique a été plutôt modifiée par cette constatation que la mastite due aux streptocoques de l'homme peut aussi apparaître chez les vaches sans association avec une maladie humaine.

Un compte rendu succinct des publications récentes montre qu'un tel fait est plus fréquent qu'on ne l'a cru.

Frost [4], résumant les constatations faites aux Etats-Unis, rapporte, en 1932, que l'examen pormanent, pendant six années, des prélèvements de mastites dans les fermes du Wisconsin et de l'Illinois, décela 58 vaches infectées par $S$. epidemicus. Des observations similaires ont été pratiquées dans quelques autres pays. Ainsi, en Allemagne, Seelemann et Hadenfeld [14] rencontrent 14 cas de mastites graves dues à $S$. pyogenes dans un troupeau de 80 vaches. En GrandeBretagne, Minett et Stableforth [8] ont isolé des streptocoques hémolytiques, ne pouvant être différenciés de $S$. pyogenes de l'homme, dans 13 cas cliniques sur 82 de mastites dues aux streptocoques.

En présence de ces observations, démontrant que la vache joue un rôle dans des épidémies étendues de maladies transmises par le lait, on peut croire que toutes les vaches recélant le streptocoque hémolytique de l'homme constituent une très effective menace pour la santé publique.

\section{LE DIAGNOSTIC DE LA MASTITE STREPTOCOCCIQUE CHRONIQUE.}

Il est bien connu que, dans cette forme de la maladie, un diagnostic uniquement basé sur les signes cliniques peut être difficile ou impossible, surtout au, stade du début [15]. Dans ces cas, un examen du lait par le laboratoire est nécessaire et les méthodes qui ont été proposées dans ce but comprennent :

a) Les méthodes directes, c'est-à-dire l'examen microscopique ou l'ensemencement des prélèvements du lait ;

b) Les méthodes indirectes, consistant dans la recherche des réactions des tissus provoquées par ces bactéries. 
On peut mentionner, parmi les méthodes indirectes :

(i) L'évaluation de la teneur du lait en cellules. L'augmentation du nombre des cellules, qui se produit en général danś la mastite, peut être décelée par la numération, par l'examen des frottís de erème ou par l'appréciation de la quantité et l'observation des caractéristiques du dépôt, après la centrifugation d'un volume déterminé de lait.

(ii) La recherche de la réaction du lait. L'augmentation de l'alcalinité, survenant quelquefois dans la mastite, peut être décelée au moyen du bromo-crésol pourpre ou d'un autre indicateur approprié.

(iii) Les analyses chimiques, comme l'épreuve de la catalase, l'évaluation des chlorures.

En thèse générale, il est évident que quelques-unes des méthodes indirectes ont le mérite de la simplicité, ce qui est un avantage appréciable lorsqu'un grand nombre d'échantillons de lait doivént être examinés. D'autre part, lors d'un seul examen, un résultat négatif $n$ 'indique pas toujours l'absence d'infection ; il faut s'attendre à des résultats intermédiaires ou douteux, et un résultat positif n'indi. que pas avec certitude que l'infection est due aux streptocoques. Un autre désavantage de ces méthodes est que, dans la pratique, elles ne peuvent s'appliquer utilement qu'à des échantillons de lait de quartiers isolés.

Comme indication, quant au degré de certitude des méthodes indirectes, on peut dire que, même lorsque l'épreuve de la centrifugation est appliquée à des échantillons provenant de quartiers isolés, il n'est pas possible, par un seul examen, de dépister plus de $70 \%$ des cas. La réaction du lait est fortement altérée dans environ $25 \%$ des cas, légèrement altérée dans $40 \%$ et non modifiée pour le reste [7]. L'épreuve de la catalase donnerait un résultat positif dans environ $70 \%$ des cas.

Quant aux méthodes directes de diagnostic, l'examen microscopique des frottis avec le dépôt du lait centrifugé est simple à pratiquer ; mais malheureusement on ne peut attendre, lors d'un seul examen, un dépistage de plus de $50 \%$ des cas. La méthode est cependant à conseiller dans des examens isolés, et elle peut être utile pour le diagnostic en général, parce qu'un résultat positif fera ressortir la nécessité d'un examen par la culture.

De toutes les méthodes recommandées pour le diagnostic, l'ensemencement du sédiment du lait centrifugé est celui qui offre le plus de sécurité, et, au début de la maladie, aucune autre méthode ne peut réussir.

Dans ee but, divers milieux de culture ont été recommandés par les différents auteurs. SemLemann [13] emploie des plaques de gélose contenant du nucléinate de sodium, sur lesquelles $S$. agalactice se développe en colonies caractéristiques, formées de longues chaînes contournées, facilement visibles à un faible grossissement. Kitmmer et HaUpt [5] recommandent les plaques de gélose-sérum-alcalialbuminate, contenant du saccharose coloré avec le bromo-crésol pourpre. Les colonies produisant sur ce milieu une coloration jaune sont réensemencées, en tubes, sur un milieu semblable, mais dans lequel le saccharose est remplacé par le raffinose. Le milieu est sélectif, en ce sens que les streptocoques fermentant le saccharose, mais non le raffinose, sont très probablement le $S$. agalactice. Toutefois, il est nécessaire de confirmer le diagnostic par le passage sur d'autres milieux. 
D'autres expérimentateurs ont utilisé la gélose au sang pour le diagnostic. Minett, Stableforth et Edwards [7] pratiquent des eultures profondes sur plaques gélose-sang de bcuf, sans addition de glucose. L'avantage de la gélose au sang est que le très petit nombre des souches les plus hémolytiques de S. agalactía sont facilement révélées, tandis que le milieu est aussi convenable pour le développement d'un nombre plus élevé de souches moins hémolytiques. Avec un peu d'expérience, un diagnostic peut souvent être établi par la seule inspection de la plaque, même lorsque quelques souillures sont développées en surface. Comme avec quelques autres milieux de culture, il sera parfois nécessaire de prélever des eolonies pour l'identification.

Dans la pratique du diagnostic de la mastite dans les troupeaux, on emploiera plus d'une méthode pour le même échantillon de lait, mais le choix du procédé reste en partie une question individuelle.

A l'Institut de recherches sur la laiterie de Kiel, la méthode suivante a été adoptée par Sezelemann pendant plusieurs années :

Les échantillons de lait sont centrifugés dans des tubes à fond arrondi et, après que les caractéristiques du sédiment ont été relevées, il est utilisé pour la préparation de frottis et pour l'ensemencement en surface sur gélose en plaques. Les frottis sont colorés avec $0,2 \%$ de bleu de toluidine en solution aqueuse et examinés sous le rapport des cellules et des bactéries. L'épreuve combinée thybromolcatalase est maintenant utilisée de préférence pour le dosage des chlorures.

Ce qui suit est l'esquisse d'une technique essayée, avec des résultats encourageants, à l'Institut de pathologie animale du Royal Veterinary College de Londres.

En premier lieu, un examen de laboratoire du lait de chaque animal du troupeau n'est pas nécessaire, parce qu'un certain nombre des animaux infectés peuvent être reconnus par l'inspection clinique, qui comprend la palpation de la glande et l'examen à l'œil nu d'une mince couche du premier lait de chaque quartier. La réaction de ce lait est relevée aussi au moyen de papiers traités par une solution de bromo-crésol pourpre. Lors de cette même visite, les particularités concernant l'âge et l'anamnèse de chaque animal, ainsi que le temps écoulé depuis le dernier vêlage seront enregistrés.

Une diminution du lait, si elle ne peut être rapportée à d'autres causes et si elle est localisée à un ou deux quartiers, sera considérée comme suspecte. Il sera prélevé, sur chaque animal soumis à cette épreuve pratique préliminaire, environ $15 \mathrm{~cm}^{3}$ de premier lait pour un examen au laboratoire. S'il s'agit d'un troupeau important, on prélève d'abord un échantillon du lait de mélange de chaque vache et, si cela est nécessaire, on procède ensuite à l'examen des échantillons des quartiers. Dans le cas de troupeaux moins nombreux, des échantillons de chaque quartier peuvent ễtre prélevés dès le début. Nulle vache n'est examinée de cette manière si le vêlage ne remonte pas au moins à cinq ou six jours. On centrifuge alors $10 \mathrm{~cm}^{3}$ de chaque prélèvement et, après avoir relevé la quantité et les caractéristiques du dépôt, des dilutions de celui-ci sont ensemencées en profondeur sur plaques de gélose-sang de boeuf. Dans cette recherche, le dernier mot appartient à la culture; mais, dans les cas où l'interprétation de la culture est difficile, la connaissance de la réaction du lait et des caractères du dépôt donne des indieations complémentaires utiles. 


\section{PROPHYLAXIE DE LA MASTITE.}

On a essayé, dans les premières parties de ce rapport, de démontrer l'importance des mesures de prophylaxie contre la mastite des vaches. Les grandes pertes qu'elle inflige à l'agriculture, son influence nocive sur la qualité du lait et le fait que la maladie peut être provoquée par des streptocoques provenant de l'homme sont des raisons qui rendent urgente la solution du problème de la prévention de cette infection. Les essais qui ont été réalisés dans ce but sont discutés ci-après, avec mention spéciale de la prophylaxie par les mesures d'hygiène.

\section{VACCINATION ET CHIMIOTHERAPIE.}

Ce sujet n'est abordé qu'en passant. Les vaceins de divers types, par exemple les cultures de types variés associés (stocks-vaccins), les autovaccins ou a vaccins d'étables ", ont été largement employés pour la prévention ou le traitement de la mastite. On a utilisé en général des microbes tués ; mais, pour les streptocoques, l'emploi de cultures vivantes a été aussi recommandé.

Les méthodes de traitement qui ont été pratiquées comprennent : la traite à fond du quartier atteint; l'injection dans les quartiers atteints de solutions antiseptiques variées; l'administration de diverses substances chimiques par la voie buccale, dans les veines, ou directement dans le tissu de la glande. Plusieurs méthodes ont été diversement associées pour un même cas.

D'après certains observateurs, de bons résultats ont été obtenus dans la prévention et le traitement de la mastite avec les vaccins, et des succès ont été enregistrés aussi après un traitement local ou général avec des substances chimiques, spécialement dans les cas au début. Il est douteux que ces conclusions soient justifiées, les symptômes disparaissant fréquemment au cours de l'évolution naturelle de la maladie, sans autre traitement que les mulsions souvent répétées. Il est certain que nous ne possédons pas jusqu'ici ia démonstration expérimentale que des animaux sains peuvent être immunisés ou qu'une guérison complète, dans le sens bactèriolo. gique. puisse être obtenue par une méthode efficace. Il est bon d'avoir cette notion présente à l'esprit lorsqu'on examine les moyens de prévention.

\section{PROPHYLAXIE PAR LES MESURES D'HYGIÉNE.}

Les mesure; générales d'hygiène suivantes sont toujours importantes : observation des règles de la propreté dans l'étable, tant en ce qui concerne les animaux que le personnel; désinfection; éloignement approprié des matières infectantes; traite complète, régulière et attentive de toutes les vaches; asséchement graduel de la mamelle; précautions contre les blessures de la mamelle et des trayons; traitement immédiat et rapide des trayons ulcérés; nettoyage efficace et stérilisation des siphons de la traite mécanique. 
L'éventualité d'une mastite provoquée par des streptocoques d'origine humaine démontre la nécessité de la stricte surveillance de la santé du personnel de l'étable. A ce point de vue, l'introduetion des machines à traire dans les grands troupeaux mérite d'être encouragée.

En ce qui concerne la prophylaxie des formes plus aiguës de la mastite streptococcique, nous manquons encore d'indications certaines, en sorte que les mesures préventives doivent se confondre avec les directives générales. On propose cependant que, là où les machines à traire sont utilisées, il soit apporté la plus grande attention à la technique de leur emploi et à ne pas laisser en place les ventouses des trayons plus longtemps qu'il n'est nécessaire.

Dans le cas de Bact. pyogenes mastitis, on a recommandé que les vaches sèches se trouvant au pâturage soient rentrées de temps á autre et que les mamelles soient examinées, afin de donner les soins appropriés. Dès le début des accidents éventuels, en été, l'application aux quartiers de produits éloignant les mouches aurait donné de bons résultats.

On a songé à la possibilité de prévenir la mastite chronique streptococcique en réglant l'ordre de la traite, de manière à ce que toutes les vaches infectées soient traites les dernières. Pour que cette méthode réussisse, il est essentiel que non seulement les cas cliniques, mais toute vache atteinte de la maladie sous une forme latente, soient dépistés. Le problème du diagnostic est donc de la plus haute importance.

Une grande proportion des animaux atteints d'infections latentes peut être décelée au premier examen de laboratoire; toutefois, les vaches étant parfois infectées dans un seul quartier et quelques animaux n'excrétant les streptocoques que de façon intermittente, il est nécessaire que les épreuves de laboratoire soient répétées à des intervalles convenables, jusqu'à ce qu'on soit relativement sûr qu'aucun animal infecté ne reste méconnu. Une seconde épreuve sera pratiquée un mois après la première et, plus tard, des examens devront être réalisés, à des intervalles de trois mois par exemple. Le lait de toutes les vaches ayant récemment vêlé et qui n'ont pas été examinées auparavant, ainsi que le lait de toutes les vaches qui viennent d'être achetées, sera éprouvé. Les introductions d'animaux nouveaux seront, autant que possible, limitées aux génisses portant leur premier veau. Les vaches gravement infectées seront immédiatemenț éliminées ; mais il n'y a pas de nécessité urgente à se débarrasser de vaches atteintes d'infection bénigne latente, si elles ont une utilité. Chez ees animaux, les signes cliniques peuvent rester stationnaires pendant de longues périodes, aussi longtemps que leur état de santé général est satisfaisant et qu'elles sont traites à fond 
régulièrement. Il semble qu'il n'y ait pas une nécessité absolue d'isoler, à l'étable ou au pâturage, les vaches infectées. Toutefois, dans la pratique, les erreurs dans l'ordre de la traite seront plus faciles à éviter si les animaux infectés sont groupés, notamment lorsqu'ils sont réunis pour la traite ; ce système doit manifestement être préféré à tout autre.

On ne voudrait pas sous-estimer les difficultés de ce mode de prophylaxie, mais on admettra que les restrictions nécessaires ne sont pas gênantes et qu'elles ne compliquent pas les précautions adoptées contre d'autres maladies contagieuses, comme la tuberculose et l'avortement épizootique. Leur mise en pratique est comparativement simple et les trayeurs s'habitueront rapidement aux nouveaux arrangements. Le succès dépendra en grande partie d'une coopération étroite avec le laboratoire, de la patience et de la persévérance du propriétaire et de l'attention avec laquelle les instructions seront appliquées. Enfin, si des examens de lait doivent être réalisés en masse, le travail devra être poursuivi dans les laboratoires qui peuvent être convenablement équipés, sans frais excessifs.

Les renseignements ne sont pas actuellement suffisants pour préciser la proportion dans laquelle on peut attendre le succès de mesures hygiéniques basées sur ces directives. Néanmoins, il n'est pas douteux que ces mesures procureront aux propriétaires un grand bénéfice financier, même si l'élimination totale de l'infection n'est pas facilement atteinte.

Durant ces dernières années, une certaine expérience a pu être acquise concernant cette prophylaxie de la mastite streptococcique. Il est évident que le progrès dépendra largement de l'étendue de l'infection originelle et que l'on ne pourra escompter un rapide succès, en général.

En collaboration avee l'Institut de recherches du Royal Veterinary College de Londres, des mesures prophylactiques sont maintenant adoptées par les propriétaires de six troupeaux, comptant au début 400 vaches environ. Il est trop tôt pour donner des renseignements quant aux résultats, mais on peut dire que la maladie paraît avoir été effectivement enrayée.

SEELEMANN [13] rapporte aussi des résultats encourageants dans deux troupeaux comptant au début 155 et 134 vaches : en dix-huit mois, les pourcentages d'infection ont été réduits de 40 à 10 et de 70 à 20 . L'application des mesures prophylactiques est poursuivie dans ces troupeaux.

\section{BIBLIOGRAPHIE.}

[1] Armstrong (C.) and Parran (T.), 1927, U.S. Pub. Health Reps., Suppl, 62, 1.

[2] Brown (J. H.), Frost (W. D.) and Shaw (M.), 1926, J. Inf. Dis, , 38, 381.

[3] Edwards (S. J.), 1932. J. Comp. Path. and Therap., 45, 43.

[4] Frost (W. D,), 1932. J. Bact. 23, 88. 
[5] Kinmmer (M.) and Haupt (H.), 1930, Erg. Hyg., 11, 354.

[6] Minett (F. C.), Stableforth (A. W.) and Edwards (S. J.), 1929. J. Comp. Path. and Therap., 42, 213.

[7] Ibid., 43, 163.

[8] Ibid., 44, 114.

[9] Ibid., 45, 1.

[10] Pröscholdt (O.), 1928, Arch. f. Tierheilk., 58, 485.

[11] Rolle (M.), 1929. Biologie des Bacterium pyogenes. M. et H. Schaper, Hanovre.

[12] Savage (W. C.), 1912. Milk and the Public Health. Macmillan and Co., London.

[13] Seelemann (M.), 1932. Die Streptokokkeninfektionen des Euters. M. et H. Schaper, Hanovre.

[14] Seelemann (M.) et Hadenfeld (A.), 1930. Zent. f. Bakt., Orig., 118, 331.

[15] Sтеск (W.), 1930. Die latente Infektion der Milchdrüse. M. et H. Schaper, Hanovre.

\title{
PROJET DE RÉSOLUTION.
}

La mastite, chez la vache, et en particulier la forme streptococcique chronique, est si répandue et d'une importance si considérable que le problème de sa prophylaxie est devenu urgent. Jusqu'à ce jour, aucun traitement chimiothérapeutique ou immunologique n'a donné de résultats vraiment satisfaisants ; mais il est déjà suffisamment démontré que les pertes causées par la mastite streptococcique chronique peuvent être considérablement réduites par les mesures sanitaires, qui n'impliquent que la séparation des vaches laitières infectées. Pour le succès de ces mesures, la première condition essentielle est que Tous les cas d'infection latente de la mamelle soient décelés. Elle nécessite l'utilisation, pour le diagnostic, de méthodes de laboratoire; mais si, comme il est à désirer, ces examens doivent être réalisés sur une large échelle, des laboratoires convenablement outillés seront nécessaires, sans que leur organisation soit nécessairement dispendieuse.

\section{LES MAIMIMITES ET LA TRAITE MÉCANIQUE}

\author{
par B. ORRE
}

de la Société Alfa-Laval, Stockholm

C'est une chose très nécessaire, pour celui qui s'occupe du développement de la traite mécanique, que de s'intéresser à la question des mammites et à l'hygiène de l'étable en général. Avec une traite hermétique, une machine bien soignée, on peut obtenir un lait de toute première qualité; mais par contre, il est clair que si le nettoyage de la machine est négligé, c'est le principe même de la traite mécanique qui est visé, et c'est cette traite que l'on blâme au sujet de la mauraise qualité du lait recueilli. 